\title{
Amplifications of the epidermal growth factor receptor gene (egfr) are common in phyllodes tumors of the breast and are associated with tumor progression
}

\author{
Christian Kersting ${ }^{1, *}$, Arno Kuijper ${ }^{2, *}$, Hartmut Schmidt ${ }^{1}$, Jens Packeisen ${ }^{1}$, \\ Cornelia Liedtke ${ }^{1}$, Nicola Tidow ${ }^{1}$, Christian Gustmann ${ }^{3}$, Bernd Hinrichs ${ }^{4}$, Pia Wülfing ${ }^{5}$, \\ Joke Tio ${ }^{5}$, Werner Boecker ${ }^{1}$, Paul van Diest ${ }^{2}$, Burkhard Brandt ${ }^{6}$ and Horst Buerger ${ }^{1}$ \\ ${ }^{1}$ Institute of Pathology, University of Muenster, Muenster, Germany; ${ }^{2}$ Department of Pathology, University \\ Medical Center Utrecht, Utrecht, The Netherlands; ${ }^{3}$ Institute of Pathology, Limburg, Germany; ${ }^{4}$ Institute of \\ Pathology, Köln-Rodenkirchen, Germany; ${ }^{5}$ Department of Obstetrics and Gynecology, University of Muenster, \\ Muenster, Germany and ${ }^{6}$ Institute of Tumour Biology, University of Hamburg, Hamburg, Germany
}

\begin{abstract}
Phyllodes tumors of the breast are rare biphasic tumors with the potential for invasion and metastatic spread. An important role of the epidermal growth factor receptor (EGFR) in phyllodes tumors has been proposed. However, detailed pathogenetic mechanisms remained unclear. We investigated 58 phyllodes tumors of the breast ( 40 benign, 10 borderline and eight malignant) by means of egfr fluorescence in situ hybridization (FISH) and gene dosage PCR for a regulatory sequence within intron 1 of egfr. Immunohistochemical staining was performed for EGFR, p16, p21, p27, p53, c-myc, Cyclin A, Cyclin D1, Cyclin E, c-kit and Ki67. Immunopositivity for EGFR was detected in $19 \%$ of phyllodes tumors ( $75 \%$ of all malignant tumors) in stromal tumor cells but not in the epithelial component. Whole-gene amplifications were seen by FISH in $15.8 \%$ (in stromal cells only) and intron 1 amplifications by gene dosage PCR in as much as $41.8 \%$ of all phyllodes tumors. Significant correlations were seen between tumor grade on the one hand and EGFR overexpression $(P=0.001)$ and intron 1 amplifications $(P<0.05)$ on the other. EGFR overexpression further correlated positively with immunohistochemical staining for p53, p16, Cyclin A, Cyclin E, Ki67 and c-kit. Presence of intron 1 amplifications correlated with p16 $(P<0.01)$, p21 $(P=0.009)$ and p53 immunoreactivity $(P<0.001)$. Neither EGFR overexpression nor whole-gene amplification was observed in a control series of 167 fibroadenomas and only one of $43(2.3 \%)$ exhibited intron 1 amplification in gene dosage PCR. In conclusion, our results show for the first time that activating mutations in and overexpression of egfr are associated with the progression in grade of phyllodes tumors of the breast. The observed association between intron 1 amplification and overexpression of EGFR provides further insight into regulation mechanisms of EGFR overexpression.

Laboratory Investigation (2006) 86, 54-61. doi:10.1038/labinvest.3700358; published online 24 October 2005
\end{abstract}

Keywords: egfr; phyllodes tumor; amplification; expression; immunohistochemistry

Phyllodes tumors are rare tumors characterized by a hypercellular monoclonal stroma and a usually modest polyclonal epithelial component, thereby representing one end of a spectrum of fibroepithelial breast tumors. ${ }^{1}$ The vast majority of phyllodes tumors are benign. However, some can also exhibit

Correspondence: Dr H Buerger, MD, PhD, Institute of Pathology, University of Münster, Albert-Schweitzer-Str. 33, 48149 Münster, Germany.

E-mail: burgerh@uni-muenster.de

*These two authors contributed equally to this work

Received 28 April 2005; revised 17 August 2005; accepted 22 August 2005; published online 24 October 2005 a malignant clinical behavior with local recurrences and distant metastatic spread. ${ }^{2}$ The histologic characteristics of the stroma determine whether a tumor is classified as benign or malignant. ${ }^{3,4}$ The spectrum of stromal morphologies is very broad, from obviously benign with only moderately cellular stroma lacking atypia and mitoses; to frankly malignant with highly cellular stroma rich in mitoses and heterologous differentiation, as seen in other soft tissue sarcomas. There are a number of histologic characteristics that have been shown to correlate with clinical outcome and thus can be helpful in grading of phyllodes tumors. ${ }^{5-10}$ However, no morphological feature has been identified so far 
that exclusively allows for definitive determination of the tumor grade by itself.

Recent studies have provided evidence for the involvement of various biological factors involved in the pathogenesis and progression of breast phyllodes tumors such as overexpression of c-myc and c-kit and a deregulated Wnt-pathway. ${ }^{11}$ Interestingly, mutations within these genes have not been observed. In other studies, involvement of the epidermal growth factor receptor (EGFR) in tumor progression has been postulated, even though egfr gene mutations have not been investigated.

In this study we aimed to gain further insight into the biology of phyllodes tumors of the breast. We assessed the overexpression of EGFR, egfr wholegene amplifications and amplification status of a short CA repeat within intron 1 of egfr, which has been shown to have regulatory impact on egfr transcription and expression. ${ }^{12,13}$ Additionally, the expression profiles of a number of markers in involved cell cycle regulation, proliferation and apoptosis were analyzed by means of tissue microarrays. We show that intron 1 amplifications within the egfr gene are quite common in phyllodes tumors and are clearly associated with overexpression of EGFR as well as grade of malignancy.

\section{Materials and methods}

A total of 58 phyllodes tumors and 167 fibroadenomas of the female breast were retrieved from the files of the Institute of Pathology, University of Muenster, the Institute of Pathology in Osnabrueck, Klinikum Osnabrueck and the Institutes of Pathology in Koeln-Rodenkirchen and Limburg, Germany. The cases were reviewed and graded according to the guidelines of the $\mathrm{WHO}^{14}$ by at least two pathologists. We were aware that no single histological feature alone will in all cases provide a universally accepted diagnosis. Therefore, we tried to appreciate the tumors' complete histological appearance as a whole utilizing the six attributes recommended by the WHO: stromal hypercellularity, cellular pleomorphism, mitosis rate, character of margins, stromal pattern and existance heterologeous stromal elements. The collection comprised eight malignant, 10 borderline and 40 benign tumors. Tumor specimens were used for investigation after informed consent. The use of tumor tissue was also approved by the local ethical committee.

Clinical follow-up data could be gathered for 15 patients with phyllodes tumors, three of which featured malignant disease. The mean follow-up period was 5.1 years, ranging from 1 to 10 years.

\section{Tissue Microarray}

A tissue microarray of 58 phyllodes tumors of the breast was constructed according to standard protocols using a dedicated TMA instrument (Beecher Instruments, Silver Spring, MD, USA). ${ }^{15,16}$ Six cores of $0.6 \mathrm{~mm}$ in diameter were punched out of the donor block and placed at a distance of $0.2 \mathrm{~mm}$ in the recipient block. By using six needle cores we even surpassed the recommendations of Hoos et $a l,{ }^{17}$ who demonstrated the sufficiency and representativity of at least three cores in TMAs for the investigation of mesenchymal tumors. For the localization of representative tumor areas including epithelial and stromal component, hematoxylin and eosin-stained sections were prepared from each original tumor block.

A second control tissue array composed of 167 fibroadenomas was constructed in a similar manner.

\section{Immunohistochemistry}

For immunohistochemical detection of EGFR, extensive testing of several antibodies was conducted. Thereby we could show considerable differences in EGFR expression frequency in soft tissue sarcomas. ${ }^{18}$ The most reliable antibody was then chosen for EGFR staining in this study. Table 1 shows the sources of the antibodies, dilutions, and antigen retrieval methods applied. For all antibodies, endogenous peroxidase activity was blocked for $30 \mathrm{~min}$ in a methanol solution containing $0.3 \%$ hydrogen peroxide after deparaffination and rehydration.

Table 1 Antibodies used for staining of phyllodes tumors

\begin{tabular}{|c|c|c|c|c|}
\hline Antibody & Source & Clone & Dilution & Pretreatment \\
\hline EGFR & Monosan & EGFR.113 & $1: 20$ & Steamer, citrate buffer, $35 \mathrm{~min}$ \\
\hline c-kit & DAKO & (polyclonal) & $1: 100$ & Steamer, citrate buffer, $35 \mathrm{~min}$ \\
\hline Ki-67 & DAKO & Mib-1 & $1: 1000$ & Steamer, citrate buffer, $35 \mathrm{~min}$ \\
\hline p53 & DAKO & DO-7 & $1: 2000$ & Steamer, citrate buffer, $35 \mathrm{~min}$ \\
\hline c-myc & Santa Cruz & 9E10 & $1: 150$ & Steamer, citrate buffer, $35 \mathrm{~min}$ \\
\hline $\mathrm{p} 16^{\text {INK4a }}$ & Neomarkers & $16 \mathrm{P} 07$ & $1: 160$ & EDTA buffer, $20 \mathrm{~min}, 97^{\circ} \mathrm{C}$ \\
\hline $\mathrm{p} 21$ & Oncogene & WAF1 & $1: 10$ & Citrate buffer, $20 \mathrm{~min}, 97^{\circ} \mathrm{C}$ \\
\hline $\mathrm{p} 27^{\mathrm{Kip} 1}$ & BD Pharmingen & 57 & $1: 1000$ & Citrate buffer, $20 \mathrm{~min}, 97^{\circ} \mathrm{C}$ \\
\hline Cyclin A & Monosan & 6E6 & $1: 100$ & Citrate buffer, $20 \mathrm{~min}, 97^{\circ} \mathrm{C}$ \\
\hline Cyclin D1 & Novocastra & DCS-6 & $1: 20$ & EDTA buffer, $20 \mathrm{~min}, 97^{\circ} \mathrm{C}$ \\
\hline Cyclin E & Monosan & 13A3 & $1: 200$ & Citrate buffer, $20 \mathrm{~min}, 97^{\circ} \mathrm{C}$ \\
\hline
\end{tabular}


After antigen retrieval, specimens were allowed to cool for $30 \mathrm{~min}$, then incubated overnight at $4^{\circ} \mathrm{C}$ with primary antibody. Before the slides were mounted, all sections were dehydrated in alcohol and xylene.

For EGFR, p16, p21, p27, Cyclin A, Cyclin D1 and Cyclin E, primary antibodies were detected using a biotinylated rabbit anti-mouse antibody (DAKO). The signal was amplified by avidin-biotin complex formation and developed with diaminobenzidine followed by haematoxylin counter staining. For EGFR, slides with an EGFR overexpressing cell line (MDA-MB-486) and one without EGFR expression (SKBR-3) were used as positive and negative controls, respectively.

For c-kit, p53 and Ki67, the primary antibody was incubated for $25 \mathrm{~min}$ at room temperature using a DAKO Autostainer instrument. The primary antibodies were detected using the LSAB/AP method (DAKO). The signal was then developed with DAKO Red, followed by haematoxylin counter staining.

For c-myc, the slides were incubated with the primary antibody for $16 \mathrm{~h}$ at $4^{\circ} \mathrm{C}$. After treatment with a rabbit anti-mouse secondary antibody, the signal was detected using the APAAP method. The slides were developed with new fuchsin (DAKO), followed by haematoxylin counter staining.

For evaluation of EGFR expression, membranous and cytoplasmic staining of tumor cells was scored from 0 to 3 ( 1 weak: at least $10 \%$ of tumor cells with a faint staining intensity; 2 moderate: at least $10 \%$ with a moderate staining intensity; 3 strong: at least $10 \%$ with a strong staining intensity). c-kit expression was graded as previously described. ${ }^{11}$ For c-kit and EGFR, cases with a score $>1$ were regarded as immunoreactive. For p16, p27, Cyclin A, Cyclin E, Ki67 and c-myc, cases with more than $10 \%$ of positively staining nuclei were regarded as immunoreactive, for p21 and Cyclin D1 cases with more than $5 \%$. For testing purposes, Ki67 staining scores were grouped from 0 to 3 . The different grading systems did not alter the significance of correlations between Ki67 and tumor grade and gene dosage PCR. p53 nuclear staining intensity was scored from 0 to 3 , whereas cases $>1$ were regarded as immunoreactive.

\section{Fluorescence In Situ Hybridization}

The probe for egfr detection was derived from homo sapiens PAC clone containing the whole egfr gene (GenBank accession no. AC006977). Nick translation was performed following standard protocols for labeling of DNA with digoxigenin-11-dUTP. For denaturation the probe was applied for $5 \mathrm{~min}$ at $70{ }^{\circ} \mathrm{C}$ to $70 \%$ formamid- $0.6 \times$ SSC. Slides underwent pretreatment with Proteinase $\mathrm{K}$ for $45 \mathrm{~min}$ at $45^{\circ} \mathrm{C}$. Hybridization to TMA sections of $4 \mu \mathrm{m}$ thickness was carried out overnight at $37^{\circ} \mathrm{C}$ in a $50 \%$ formamid- $1 \times$ SSC- $10 \%$ dextran sulfate solution in the presence of Cot-1-DNA (Life Technologies, Inc.,
Carlsbad, USA) and HPL-DNA (Sigma, St Louis, USA). Pos-hybridization washes were performed at $45^{\circ} \mathrm{C}$ in $50 \%$ formamide- $2 \times$ SSC and $0.1 \times$ SSC at $60^{\circ}$ followed by blocking with $3 \%$ BSA in $4 \times$ SSC at $37^{\circ} \mathrm{C}$. Probes were detected using mouse-anti-digoxigenin (Sigma, St Louis, USA) and Cy3-labeled goatantimouse antibodies (Dianova $\mathrm{GmbH}$, Hamburg, Germany) for $45 \mathrm{~min}$ each at $37^{\circ} \mathrm{C}$.

For each core, 20 nuclei were selected for scoring according to morphological criteria using DAPI counterstaining. Only nonoverlapping, intact nuclei were scored. Clearly distinguishable non-tumor cells were disregarded. The cutoff for considering a case as amplified was set at 4 signals per nucleus. Scoring was performed as previously published. ${ }^{19,20}$

\section{Gene Dosage PCR}

DNA was extracted from five whole paraffin sections of tumor tissue with a thickness of $10 \mu \mathrm{m}$ each according to standard protocols. Furthermore, 10 samples of normal appearing breast tissue surrounding the phyllodes tumors were analyzed.

For detection of amplifications of the first CA repeat in the first intron of the egfr gene, a quantitative real-time PCR was performed targetting the repeat, and two known single-copy genes, ${ }^{21}$ superoxide dismutase (sod) and hemoglobin beta $(h b b)$ genes, as reference.

Specific primers for sequences flanking the first CA repeat in the first intron of the egfr gene were designed (CAIfor: $5^{\prime}$-tgaagaatttgagccaaccaaa- $3^{\prime}$ and CAIrev: $5^{\prime}$-cacttgaaccagggacagca-3') using Primer Express software (Applera). They were chosen since previous studies demonstrated that this primer combination defines amplifications of the whole gene, amplifications restricted to the CA-SSR I repeat and mutations involving this polymorphic sequence. Also a universal, VIC labeled probe consisting of 15 CA repeats (minor groove binder probe: nine repeats) was constructed. The primers were checked by BLAST search (Internet address: http://www.ncbi.nlm.nih.gov/Sitemap/index.html\# BLAST) and represented specific sequences for egfr. Primers and probes were also designed for the single-copy genes sod2 (chromosome 6q25, GenBank accession no. 65965, forward primer: 5'-GGAGAAGCTGACGGCTGC-3', reverse primer: $5^{\prime}$-CCTTATTGAAACCAAGCCAACC-3', VIC-labeled probe: 5'-CAACCTGAGCCTTGGACACCAACAGA-3') and $h b b$ (chromosome 11p, GenBank accession no. V00499, forward primer: 5'-GTGAAGGCTCATGGC AAGAAAG-3', reverse primer: 5'-CAGCTCACTCAG TGTGGCAAAG-3', VIC-labeled probe: ATGGCCTG GCTCACCTGGACAACC). The amplicon length was minimized (68-97 bp) for all three of the genes, to allow for the most efficient PCR amplification. PCR analysis was performed using TaqMan Universal Mix (Applera) and detection was performed on an ABI Prism 7900HT Sequence Detection System (Applera). Cycling conditions were as follows: 
denaturation: $95^{\circ} \mathrm{C}, 15 \mathrm{~s}$, annealing and extension $60^{\circ} \mathrm{C}, 1 \mathrm{~min}$, using 40 cycles. All PCR reactions were performed in triplicate and in at least two independent reactions. Serial dilutions of DNA were used to ensure accuracy of gene dosage quantification. The copy number of the egfr gene was measured in the breast cancer-derived cell line MDA-MB-468 in comparison with normal leukocytes. MDA-MB-468 DNA reportedly displays a 30-50-fold amplification of the egfr gene and was used as a positive control. DNA concentrations were normalized to both sod2 and $h b b$.

\section{Statistical Analysis}

Statistical analysis and tests were performed with SPSS Version 11.5.1. Correlations between EGFR expression, amplification and clinicopathological features were tested with cross tables applying $\chi^{2}$ and correlation analysis was performed according to Kendall (Tau b).

\section{Results}

Of the 58 phyllodes tumors, 57 could be analyzed by FISH, 55 by gene dosage PCR, 57 by immunohistochemistry for c-kit, 54 for Cyclin D1 and 52 for p21. For all other markers, all cases could be analyzed.

Significant differences could be seen in the reaction patterns between stroma and epithelium. The epithelial component mostly displayed a reaction pattern as seen in the normal breast (data not shown). Therefore only data on the stromal compartment are presented.

\section{EGFR Expression}

As shown in Table 2, EGFR overexpression was detected in $19 \%$ of all tumors: in $12.5 \%$ of benign, in $10 \%$ of borderline and $63 \%$ of all malignant phyllodes tumors $(P=0.001)$. EGFR overexpression was almost exclusively restricted to stromal cells (Figure 1). Epithelial cells stained weakly positive in only two cases of benign phyllodes tumors. Stromal cells usually featured a combined staining of membranes and cytoplasm. Expression of EGFR was often observed in myothelial cells but not consistently. Overexpression of EGFR correlated significantly positively with the expression of p53 $(P=0.001)$, p16 $(P<0.05)$, c-kit $(P<0.01)$, Ki67 $(P<0.05)$, Cyclin A $(P<0.01)$ and Cyclin E $(P<0.05)$ (see Table 3). A trend towards statistical significance could be seen for p21 and c-myc.

\section{Amplifications of a Regulatory Sequence within Intron 1 of egfr}

Amplifications of the CA repeat within (Figure 2) intron 1 were seen in 23/55 (41.8\%) of all cases and
Table 2 EGFR overexpression and egfr amplification in breast phyllodes tumors of different grade

\begin{tabular}{|c|c|c|c|c|c|c|}
\hline \multirow[t]{2}{*}{ Type of egfr amplification } & \multicolumn{2}{|c|}{ Benign } & \multicolumn{2}{|c|}{ Borderline } & \multicolumn{2}{|c|}{ Malignant } \\
\hline & - & + & - & + & - & + \\
\hline Whole gene & 36 & 3 & 9 & 1 & 6 & 2 \\
\hline Intron 1 CA repeat & 23 & 15 & 7 & 2 & 2 & 6 \\
\hline \multicolumn{7}{|l|}{ Overexpression } \\
\hline Stromal & 35 & 5 & 9 & 1 & 3 & 5 \\
\hline Epithelial & 31 & 2 & 8 & 0 & 3 & 0 \\
\hline
\end{tabular}
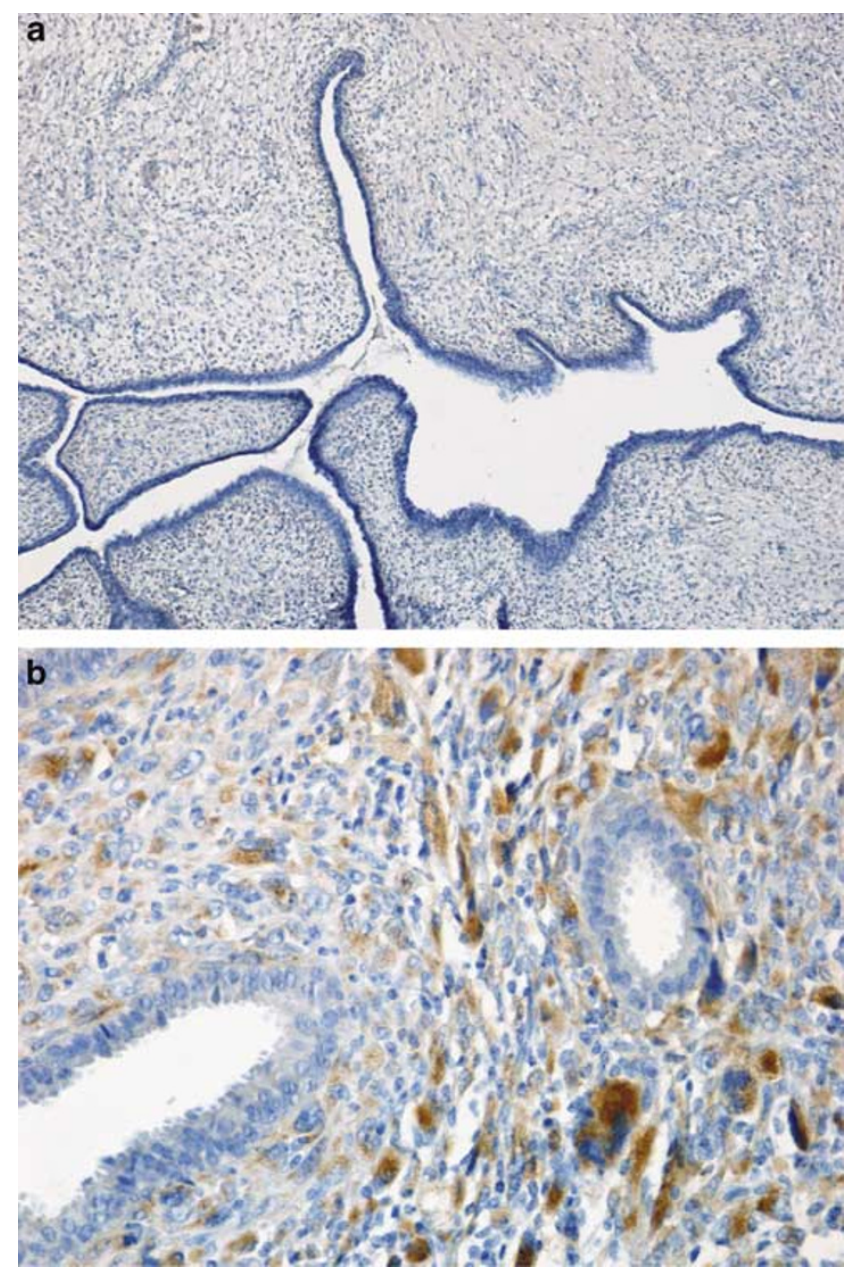

Figure 1 Photomicrographs of a phyllodes tumor without (a) and with EGFR immunopositivity (b). Noteworthy, the epithelial cell compartment lacks EGFR immunoreactivity $(\mathbf{a} \times 10, \mathbf{b} \times 20)$.

were associated with tumor grade $(P<0.05)$ (see Table 2). This included 15/38 benign, $2 / 9$ borderline and 6/8 malignant phyllodes tumors. The average gene copy number was 5.2, the maximum observed copy number was 22.9 in one sample. Intron 1 CA repeat amplifications correlated significantly positively with expression of EGFR $(P<0.01)$, p53 
Table 3 Comparison between two types of egfr amplification, EGFR expression and several biomarkers in breast phyllodes tumors

\begin{tabular}{|c|c|c|c|c|c|c|c|c|c|c|c|c|c|c|c|c|c|c|c|c|}
\hline \multirow[t]{2}{*}{ Type of egfr detection } & \multicolumn{2}{|c|}{ p16 } & \multicolumn{2}{|c|}{$p 21$} & \multicolumn{2}{|c|}{$p 27$} & \multicolumn{2}{|c|}{ p53 } & \multicolumn{2}{|c|}{ Cyclin A } & \multicolumn{2}{|c|}{ Cyclin D1 } & \multicolumn{2}{|c|}{ Cyclin E } & \multicolumn{2}{|c|}{$c$-kit } & \multicolumn{2}{|c|}{$c-m y c$} & \multicolumn{2}{|c|}{$K i-67$} \\
\hline & - & + & - & + & - & + & - & + & - & + & - & + & - & + & - & + & - & + & - & + \\
\hline \multicolumn{21}{|l|}{ Whole gene } \\
\hline- & 13 & 38 & 18 & 28 & 4 & 47 & 46 & 5 & 48 & 3 & 37 & 10 & 43 & 8 & 45 & 5 & 34 & 16 & 41 & 10 \\
\hline+ & 2 & 4 & 0 & 5 & 0 & 6 & 3 & 3 & 5 & 1 & 6 & 0 & 3 & 3 & 4 & 2 & 2 & 4 & 3 & 3 \\
\hline \multicolumn{21}{|l|}{ Intron 1 repeat } \\
\hline- & 4 & 28 & 14 & 16 & 4 & 28 & 32 & 0 & 32 & 0 & 25 & 5 & 27 & 5 & 30 & 2 & 19 & 13 & 27 & 5 \\
\hline+ & 11 & 12 & 2 & 17 & 0 & 23 & 15 & 8 & 19 & 4 & 16 & 5 & 17 & 6 & 17 & 5 & 16 & 7 & 15 & 8 \\
\hline \multicolumn{21}{|l|}{ Expression } \\
\hline- & 10 & 37 & 18 & 24 & 4 & 43 & 44 & 3 & 46 & 1 & 35 & 8 & 41 & 6 & 43 & 3 & 32 & 14 & 39 & 8 \\
\hline+ & 6 & 5 & 1 & 9 & 0 & 11 & 6 & 5 & 8 & 3 & 9 & 2 & 6 & 5 & 7 & 4 & 5 & 6 & 6 & 5 \\
\hline
\end{tabular}
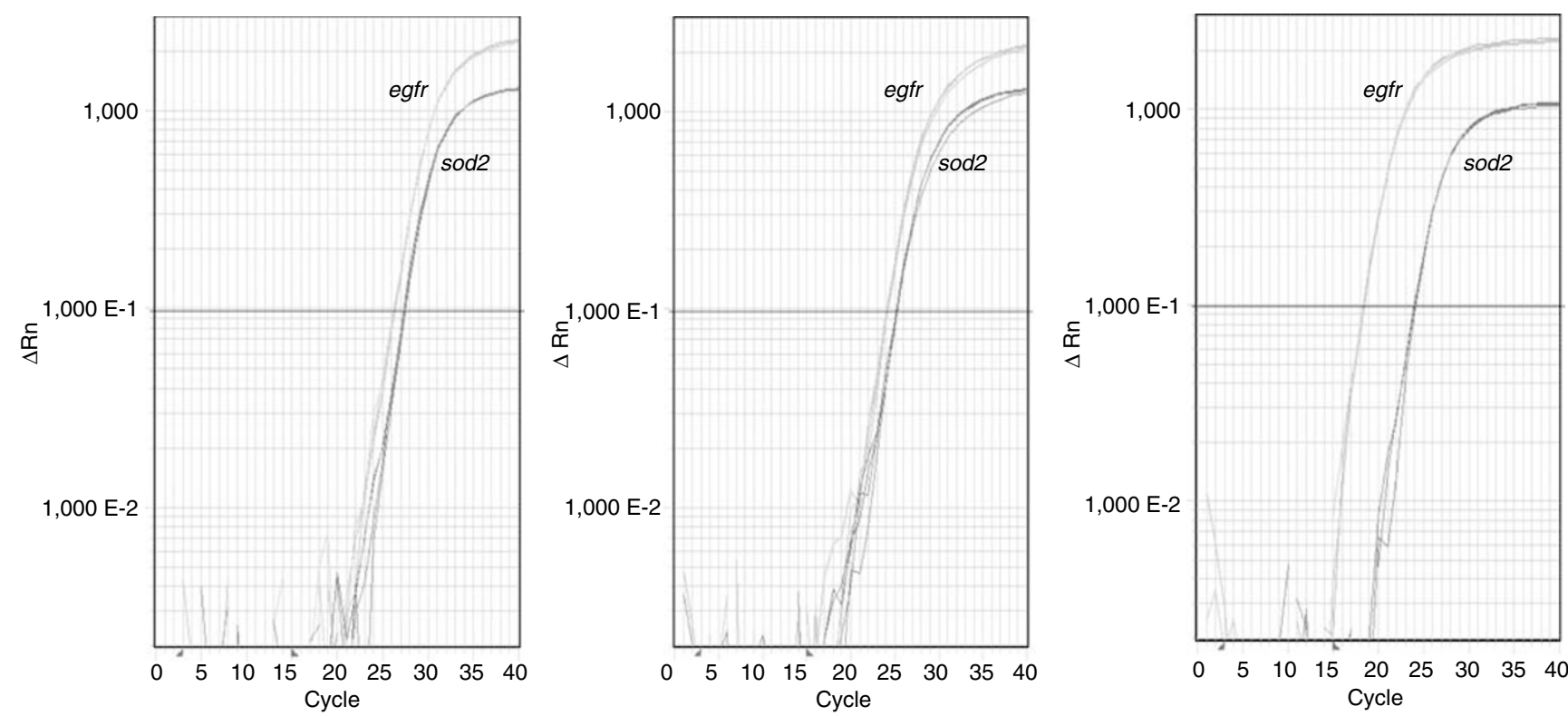

Figure 2 Diagram of gene dosage PCR showing elevated egfr amplicon concentration of a phyllodes tumor sample with high level amplification (right) compared to a sample without amplification (middle) and a negative control (left).

$(P<0.001)$, Cyclin A $(P<0.05)$, p16 $(P<0.05)$ and p21 $(P<0.01)$ (see Table 3).

Normal appearing breast tissue surrounding phyllodes tumors showed intron 1 amplifications in two of 10 cases.

\section{FISH Analysis}

egfr whole-gene amplifications detected by FISH were present in only $15.8 \%$ of all cases (Tables 2 and 3 ): in $3 / 39$ benign, $1 / 10$ borderline and $2 / 8$ malignant tumors. Whole-gene amplifications occured exclusively in stromal cells (Figure 3), two of which were high level ( $>10$ copies per nucleus), while the other four were low-level amplifications. When amplification was observed, the majority of cells was affected. Whole-gene amplifications did not correlate with tumor grade (see Table 2). A positive correlation could be observed with expression of EGFR $(P<0.001)$, p53 $(P<0.01)$ and Cyclin E $(P<0.05)$, though (see Table 3$)$.

\section{Comparison between FISH and Gene Dosage PCR}

Whole-gene amplification and intron 1 CA repeat amplification of egfr were significantly correlated with each other $(P<0.01)$ and with EGFR overexpression $(P<0.01$ for the intron 1 CA repeat amplifications; $P<0.001$ for whole-gene amplifications), but observed frequencies differed clearly. Although all tumors with egfr whole-gene amplifications in FISH displayed amplifications in gene dosage PCR, only nine out of 23 tumors with CA repeat amplification in gene dosage PCR also featured whole-gene amplifications in FISH (Table 4). 

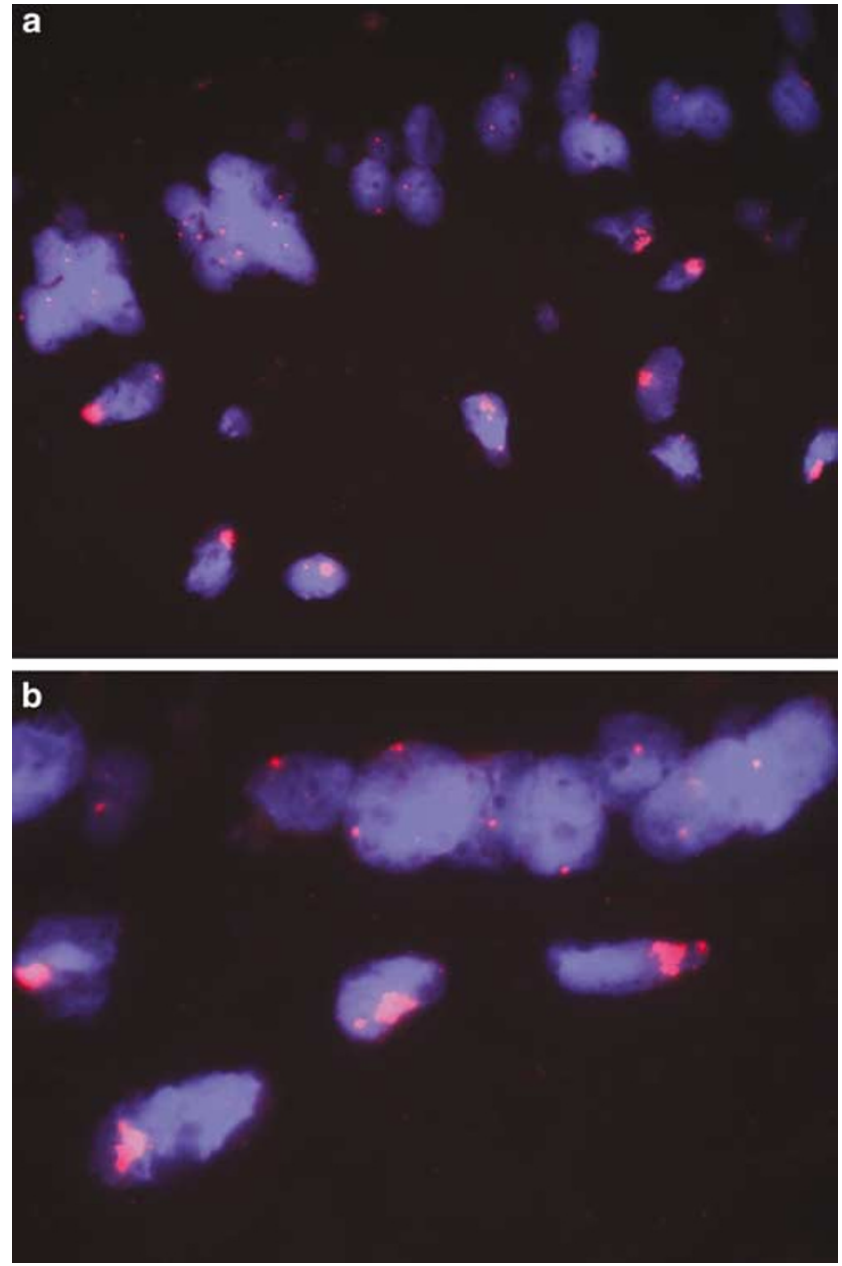

Figure 3 Fluorescence images of two cases of malignant phyllodes tumor showing stromal tumor cells with egfr whole-gene amplifications below a line of epithelial cells with regular number of gene copies $(\mathbf{a} \times 20, \mathbf{b} \times 40)$.

Table 4 Comparison between two types of egfr amplification in breast phyllodes tumors

Whole gene amplification Total

$-$

\begin{tabular}{llll}
\hline Intron 1 amplification & & & \\
- & 31 & 0 & 31 \\
+ & 17 & 6 & 23 \\
Total & 48 & 6 & 54 \\
\hline
\end{tabular}

\section{Clinico-Pathological Correlations}

EGFR overexpression $(P=0.001)$ and also Cyclin A $(P<0.05)$, Cyclin E $(P<0.05)$, Ki67 $(P<0.01)$, c-kit $(P=0.001)$ and p53 $(P<0.001)$ immunoreactivity significantly correlated with grade of phyllodes tumors. None of the patients with positivestaining for the above markers for which clinical follow-up
Epidermal growth factor receptor in phyllodes tumors of the breast C Kersting et al

data were available showed recurrence or metastases

(12 benign, three malignant).

\section{Fibroadenomas}

All 167 fibroadenomas lacked immunopositivity for EGFR. Furthermore, no case with increase of egfr whole gene copy number detectable by FISH could be found. Only one (of 43 tested) revealed an amplification of the CA repeat in intron 1 of egfr by means of gene dosage PCR $(2.3 \%)$.

\section{Discussion}

The pathogenesis of breast phyllodes tumors is poorly understood. In particular, the literature concerning molecular changes within these tumors is sparse. Up to now, only correlations between expression of p53, Ki67, c-kit, PDGF, VEGF and CD10 with tumor grade ${ }^{9,22-25}$ have been described. The aim of this study was to expand on these data, especially with regard to other cell cycle regulators and the putative role of EGFR amplification by FISH and gene dosage PCR and immunohistochemistry on TMAs. As we were able to confirm the data by Chan et $a l^{22}$ and Chen et $a l^{23}$ on p53, Ki67 and c-kit, it is rather unlikely that the use of TMAs has biased our results. Unfortunately, clinical data were obtainable in only 15 of 58 cases, so our follow-up information can only be regarded as a small sample.

Our findings show for the first time whole gene and intron 1 CA repeat egfr amplifications and overexpression of EGFR in the stromal component of breast phyllodes tumors. In view of the strong correlations between amplification of the regulatory repeat in intron 1 of egfr and EGFR overexpression on the one hand and tumor grade on the other, these changes are likely to be involved in the pathogenesis and progression of the stromal component of phyllodes tumors. This is supported by our findings in normal breast tissue surrounding the tumors, revealing low-level amplifications of the intron $1 \mathrm{CA}$ repeat in $20 \%$ of the cases. An association between EGFR overexpression and tumor progression has been suggested earlier. ${ }^{26}$ However, we are now able to show that amplifications of genetic sequences covering important regulatory sequences of egfr and to a lesser extent of the whole egfr gene may underlie the overexpression of EGFR in the stromal component of phyllodes tumors. Unfortunately, as of today, we were not able to perform a complete sequence analysis of the intron 1 CA repeat region, although considerable efforts have been made. It seems that the nearby fragile site and the length of the repeat itself including its influence on secondary DNA structure ${ }^{12}$ contribute to the difficulties encountered.

The correlation between EGFR overexpression and small amplifications in breast cancer has been described earlier by our group. ${ }^{13}$ However, the 
60

relationship between both features is significantly higher in phyllodes tumors compared to invasive cancer of the breast. Whereas only $25 \%$ of EGFR overexpressing invasive breast cancer cases was associated with any kind of egfr amplifications, ${ }^{20}$ $82 \%$ of EGFR-overexpressing phyllodes tumors displayed these amplifications. It further seems that involvement of EGFR at the DNA and protein level is a rather specific finding for phyllodes tumors and not a general event in biphasic tumors of the breast: only one of 43 fibroadenomas featured intron 1 repeat amplifications (and no whole-gene amplification in 163 fibroadenomas), but a considerable percentage of benign and an even higher percentage of malignant phyllodes tumors did. This might give further support to the hypothesis that EGFR is part of a mechanism involved in transition from fibroadenoma towards phyllodes tumors as proposed earlier. ${ }^{1}$ It seems more likely, on the other hand, that the EGFR pathway is often activated after successful progression to phyllodes tumor and is one important factor that promotes malignant transformation, among others.

Our results further stress the importance of genetic instability in the pathogenesis of these tumors. Phyllodes tumors of the breast are frequently found in patients with Li-Fraumeni syndrome caused by p53 germline mutations and consequently cytogenetic instability. ${ }^{27}$ A DNA 'fragile site' has been described nearby the egfr locus, ${ }^{13}$ which might be a possible explanation for the rather high frequency of egfr amplifications in sporadic phyllodes tumors. Consequently, the association of p53 and p21 with increased egfr gene dosage could be regarded as downstream events due to DNA double-strand breaks and insufficient repair mechanisms. ${ }^{28}$ However, it is obvious that these mechanisms are rather weak and are directly counterbalanced by an increased proliferation rate as indicated by the correlation of Ki67, Cyclin A and E with EGFR overexpression. In conclusion, the protein expression patterns in phyllodes tumors due to egfr mutations can generally be divided into factors associated with EGFR overexpression and proliferation (Ki67, Cyclin A, Cyclin E, p16) and factors associated with counterbalancing, nevertheless finally insufficient cellular proapoptotic mechanisms due to genetic instability per se (p21).

Unfortunately, our results give only limited insight into the relationship between different types of egfr amplifications. We examined two types of egfr amplifications: more common amplifications of a regulatory CA short sequence repeat in intron 1 by gene dosage PCR and rarer amplifications of the whole gene, detectable by FISH analysis. ${ }^{29}$ The frequency of CA repeat amplifications (41\%) and whole-gene amplifications $(15 \%)$ point towards a sequence of genetic instability either way. Nevertheless, a dynamic process in the development of egfr amplifications has not been demonstrated so far and it remains to be elucidated if different kinds of egfr amplifications occur in a stepwise manner.

However, it must be stated that less than half of all phyllodes tumors revealed egfr amplifications and even less displayed an EGFR overexpression. So, EGFR is not the only pathway that promotes growth and progression in phyllodes tumors. Recent studies focused on the Wnt pathway and its possible interaction with c-myc in the pathogenesis of phyllodes tumors. However, the underlying mechanisms for c-myc deregulation in this tumor entity remained unclear, since no activating mutations could be found for the majority of tumors with overexpression of c-myc. Published data so far point towards a direct relationship between EGFR overexpression and c-myc expression. Nevertheless, our results revealed only a statistical trend to support these experimental findings and may give only a limited explanation for c-myc overexpression. ${ }^{11}$

In summary, we show for the first time that amplifications of egfr are common and correlate with EGFR overexpression as well as tumor grade, implicating egfr in progression in phyllodes tumors of the breast. As new additional markers for malignancy in phyllodes tumors they may therefore also aid in establishment of the correct diagnosis in questionable cases. Since only one whole-gene amplification of egfr was evident in our series of fibroadenomas $(2.3 \%)$ without EGFR overexpression, EGFR overexpression and amplifications allow for a quite clear distinction of both entities in difficult cases.

\section{Acknowledgements}

We thank Mrs Petra van der Groep, Ulrike Neubert and Petra Meier for help with the immunohistochemical staining and FISH analysis.

\section{References}

1 Kuijper A, Buerger H, Simon R, et al. Analysis of the progression of fibroepithelial tumours of the breast by PCR-based clonality assay. J Pathol 2002;197:575-581.

2 Hajdu SI, Espinosa MH, Robbins GF. Recurrent cystosarcoma phyllodes: a clinicopathologic study of 32 cases. Cancer 1976;38:1402-1406.

3 Sawhney N, Garrahan N, Douglas-Jones AG, et al. Epithelial-stromal interactions in tumors: a morphologic study of fibroepithelial tumors of the breast. Cancer 1992;70:2115-2120.

4 Noguchi S, Motomura K, Inaji $\mathrm{H}$, et al. Clonal analysis of fibroadenoma and phyllodes tumor of the breast. Cancer Res 1993;53:4071-4074.

5 Norris HJ, Taylor HB. Relationship of histologic features to behavior of cystosarcoma phyllodes. Analysis of ninety-four cases. Cancer 1967;20: 2090-2099.

6 Shabalova IP, Chemeris GJ, Ermilova VD, et al. Phyllodes tumour: cytologic and histologic presentation of 22 cases, and immunohistochemical demonstration of p53. Cytopathology 1997;8:177-187. 
7 Hawkins RE, Schofield JB, Fisher C, et al. The clinical and histologic criteria that predict metastases from cystosarcoma phyllodes. Cancer 1992;69:141-147.

8 Cohn-Cedermark G, Rutqvist LE, Rosendahl I, et al. Prognostic factors in cystosarcoma phyllodes. A clinicopathologic study of 77 patients. Cancer 1991; 68:2017-2022.

9 Feakins RM, Wells CA, Young KA. Platelet-derived growth factor expression in phyllodes tumors and fibroadenomas of the breast. Hum Pathol 2000;31:1214-1222.

10 Barth Jr RJ. Histologic features predict local recurrence after breast conserving therapy of phyllodes tumors. Breast Cancer Res Treat 1999;57:291-295.

11 Sawyer EJ, Poulsom R, Hunt FT, et al. Malignant phyllodes tumours show stromal overexpression of c-myc and c-kit. J Pathol 2003;200:59-64.

12 Gebhardt F, Burger H, Brandt B. Modulation of EGFR gene transcription by secondary structures, a polymorphic repetitive sequence and mutations-a link between genetics and epigenetics. Histol Histopathol 2000;15:929-936.

13 Tidow N, Boecker A, Schmidt H, et al. Distinct amplification of an untranslated regulatory sequence in the egfr gene contributes to early steps in breast cancer development. Cancer Res 2003;6:1172-1178.

14 Tavassoli FA, Devilee P (eds). World Health Organization Classification of Tumors. Pathology and Genetics of Tumours of the Breast and Female Genital Organs. IARC Press: Lyon, 2003.

15 Packeisen J, Korsching E, Herbst H, et al. Demystified...tissue microarray technology. Mol Pathol 2003;56:198-204.

16 Kononen J, Bubendorf L, Kallioniemi A, et al. Tissue microarrays for high-throughput molecular profiling of tumor specimens. Nat Med 1998;4:844-847.

17 Hoos A, Urist MJ, Stojadinovic A, et al. Validation of tissue microarrays for immunohistochemical profiling of cancer specimens using the example of human fibroblastic tumors. Am J Pathol 2001;158:1245-1251.

18 Kersting C, Packeisen J, Leidinger B, et al. Pitfalls in immunohistochemical assessment of EGFR-expression in soft tissue sarcomas. J Clin Pathol, in press.
19 Steidl C, Simon R, Burger H, et al. Patterns of chromosomal aberrations in urinary bladder tumours and adjacent urothelium. J Pathol 2002;198:115-120.

20 Kersting C, Tidow N, Schmidt H, et al. Gene dosage PCR and fluorescence in situ hybridization reveal low frequency of egfr amplifications despite protein overexpression in invasive breast carcinoma. Lab Invest 2004;84:582-587.

21 Brandt B, Vogt U, Harms F, et al. Double-differential PCR for gene dosage estimation of erbB oncogenes in benign and cancer tissues and comparison to cellular DNA content. Gene 1995;159:29-34.

22 Chan YJ, Chen BF, Chang CL, et al. Expression of p53 protein and Ki-67 antigen in phyllodes tumor of the breast. J Chin Med Assoc 2004;67:3-8.

23 Chen CM, Chen CJ, Chang CL, et al. CD34, CD117, and actin expression in phyllodes tumor of the breast. J Surg Res 2000;94:84-91.

24 Tse GM, Lui PC, Lee CS, et al. Stromal expression of vascular endothelial growth factor correlates with tumor grade and microvessel density in mammary phyllodes tumors: a multicenter study of 185 cases. Hum Pathol 2004;35:1053-1057.

25 Tse GM, Putti TC, Lui PC, et al. Increased c-kit (CD117) expression in malignant mammary phyllodes tumors. Mod Pathol 2004;17:827-831

26 Suo Z, Nesland JM. Phyllodes tumor of the breast: EGFR family expression and relation to clinicopathological features. Ultrastruct Pathol 2000;24: 371-381.

27 Birch JM, Alston RD, McNally RJ, et al. Relative frequency and morphology of cancers in carriers of germline TP53 mutations. Oncogene 2001;20: 4621-4628.

28 Gartel AL, Tyner AL. The role of the cyclin-dependent kinase inhibitor p21 in apoptosis. Mol Cancer Ther 2002;1:639-649.

29 Buerger H, Packeisen J, Boecker A, et al. Allelic length of a CA dinucleotide repeat in the egfr gene corre lates with the frequency of amplifications of this sequence-first results of an inter-ethnic breast cancer study. J Pathol 2004;203:545-550. 